If

GPO PRICE

$\mathbf{\$}$

CFSTI PRICE(S) $\$$

Hard copy $(H C) \frac{2,50}{.65}$

Microfiche (MF)

NASA

X-612-67-584

PREPRINT

ff 653 July 65

\title{
THE INTER-RELATIONSHIP OF
}

COSMIC RAY ANISOTROPIES AND THE INTERPLANETARY MAGNETIC FIELD

\section{K. G. MCCRACKEN}

U. R. RAO

N. F. NESS

NOVEMBER 1967

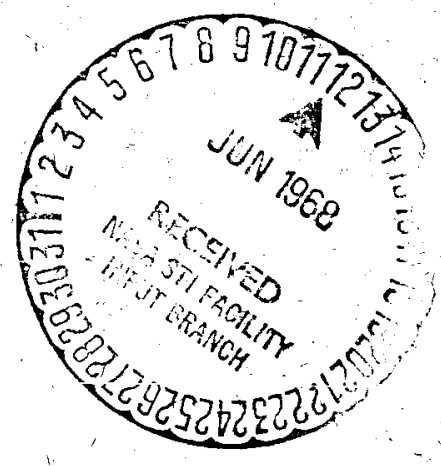

GSFC

GODDARD SPACE FLIGHT CENTER GREENBELT, MARYLAND 
THE INTER-RELATIONSHIP OF COSMIC RAY ANISOTROPIES AND THE INTERPLANETARY

MAGNETIC FIELD

\section{By}

K. G. McCracken *

U. R. Rao ${ }^{\dagger}$

N. F. Ness ${ }^{\ddagger}$

November 1967

\footnotetext{
*Department of Physics, University of Adelaide, Adelaide, South Australia

†Physical Research Laboratory, Ahmedabad, India

‡Goddard Space Flight Centre, Greenbelt, Maryland
} 
TABLE OF CONTENTS

$\underline{\text { Page }}$

ABSTRACT $\ldots \ldots \ldots \ldots \ldots \ldots \ldots \ldots \ldots \ldots \ldots \ldots \ldots \ldots$

INTRODUCTION $\ldots \ldots \ldots \ldots \ldots \ldots \ldots \ldots \ldots \ldots \ldots \ldots \ldots$

The Equilibrium Anisotropy $\ldots \ldots \ldots \ldots \ldots \ldots \ldots \ldots$

The Non-Equilibrium Anisotropy $\ldots \ldots \ldots \ldots \ldots \ldots \ldots$

THE ANISOTROPY VERSUS FIELD CORRELATION $\ldots \ldots \ldots \ldots .2$

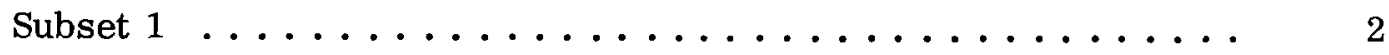

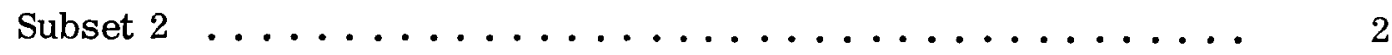

ANOMALOUS COSMIC RAY FLOW CHARACTERISTICS . . . . . . 8

CONCLUSIONS $\ldots \ldots \ldots \ldots \ldots \ldots \ldots \ldots \ldots \ldots \ldots \ldots \ldots$

The Equilibrium Anisotropy $\ldots \ldots \ldots \ldots \ldots \ldots \ldots \ldots$

The Non-Equilibrium Anisotropy $\ldots \ldots \ldots \ldots \ldots \ldots . \ldots 14$

ACKNOWLEDGEMENT $\ldots \ldots \ldots \ldots \ldots \ldots \ldots \ldots \ldots \ldots$

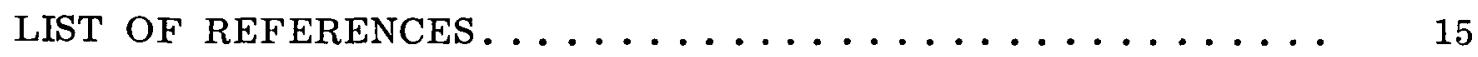

FIGURE CAPTIONS

$\underline{\text { Figure }}$

Page

1 Illustrating the difference between the equilibrium and non-equilibrium classes of cosmic ray anisotropy. The amplitudes and azimuths of the mean anisotropy for each hour are plotted as a vector addition diagram. Note definition of $\phi_{c} . \ldots \ldots \ldots \ldots \ldots \ldots \ldots \ldots \ldots \ldots \ldots \ldots$

2 The scatter diagram between the hourly means of the azimuth of the magnetic field vector and the azimuth of the equilibrium cosmic ray anisotropy. The magnetic field azimuth, $\phi_{\mathrm{m}}$, is measured east of the sun (E) while 
the cosmic ray azimuth, $\phi_{c}$, indicates the direction west of the sun (W) from which the maximum cosmic ray flux is observed: if colinear, $\phi_{\mathrm{c}}=360^{\circ}-\phi_{\mathrm{m}}$. ......

3 The scatter diagram between the hourly means of the azimuth of the magnetic field vector and the azimuth of the non-equilibrium cosmic ray anisotropy. The predicted relationship between $\phi_{\mathrm{c}}$ and $\phi_{\mathrm{m}}$ for $\eta=3.0$ is superposed on the diagram. Note that the line of best fit to the data points has a slope of less than unity. .....

4 The working model for the inter-relationship between the equilibrium anisotropy vector $\left(A_{\vec{E}}\right)$, the non-equilibrium anisotropy vector $\left(A_{\vec{N}}\right)$ directed parallel to the interplanetary magnetic field, and the observed anisotropy vector $\left(\mathrm{A}_{\overrightarrow{\mathrm{T}}}\right) . \ldots \ldots \ldots \ldots$

5 The scatter diagram between the magnetic and the cosmic ray azimuth for all the solar cosmic ray data under

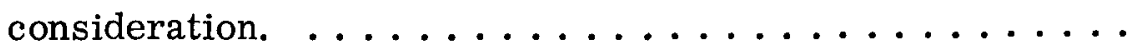

The temporal dependence of the flux of 7.5-45 Mev protons, of the azimuth of the magnetic field, and of the cosmic ray anisotropy, during the passage of a magnetic sector boundary past the spacecraft. Note the high degree of correlation between the changes in the magnetic and cosmic ray azimuths..............

$7 \quad$ Illustrating one model proposed to explain the simultaneous reversals of the magnetic and cosmic ray anisotropy azimuths. The positions of the Pioneer VI spacecraft relative to the interplanetary magnetic field at times prior, and subsequent to $0300 \mathrm{UT}$ are shown. ...

8 The detailed temporal dependence of the amplitude and azimuth of the cosmic ray anisotropy during the field and anisotropy reversal indicated in Fig. 6 by the crosshatched box. Note the abrupt nature of the reversals of the anisotropy azimuth. ................ 
THE INTER-RELATIONSHIP OF COSMIC RAY

ANISOTROPIES AND THE INTERPLANETARY

MAGNETIC FIELD

By

K. G. McCracken*

U. R. $\mathrm{RaO}^{\dagger}$

N. F. Ness $\ddagger$

\begin{abstract}
The direction of the cosmic ray anisotropy during solar proton events and the concurrent direction of the interplanetary magnetic field vector are compared using data obtained in 1966 by the Pioneer VI spacecraft. It is shown that the non-equilibrium cosmic ray anisotropy (normally observed to exhibit an amplitude in the range $20-50 \%$ ) is field aligned, while the equilibrium anisotropy (normally observed to exhibit an amplitude in the range 5-15\%) is independent of the direction of the magnetic field vector. Two anomalous proton events during which pronounced anisotropies were observed with their maximum cosmic ray fluxes directed towards the sun are discussed. It is shown that during these proton events there were synchronous reversals of $180^{\circ}$ in the direction of the magnetic field vector, and the cosmic ray anisotropy direction, in association with the passage of a magnetic sector boundary past the spacecraft. These observations are interpreted as possible evidence for their being a complex loop in the magnetic field.
\end{abstract}

\footnotetext{
*Department of Physics, University of Adelaide, Adelaide, South Australia.

†Physical Research Laboratory, Ahmedabad, India.

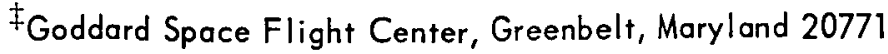




\section{THE INTER-RELATIONSHIP OF COSMIC RAY ANISOTROPIES AND THE INTERPLANETARY MAGNETIC FIELD}

\section{INTRODUCTION}

The cosmic radiation observed subsequent to the release of energetic particles by a solar flare exhibits marked departure from isotropy. In a recent paper (McCracken, Rao and Bukata, 1967, hereafter called Paper 1) it has been suggested that there are in fact two distinctly different classes of anistropy evident during cosmic ray flare effects, these being:-

(a) The Equilibrium Anisotropy

Evident at late times during a flare effect, and characterized by temporal invariance of both the anisotropy amplitude (5 to $15 \%$ ) and phase (the maximum cosmic ray flux always being from the direction of the sun). Indirect arguments have suggested that the characteristics of this anisotropy are not dependent upon the detailed nature of the interplantary magnetic field (Paper 1).

(b) The Non-Equilibrium Anisotropy

Evident at early times during a flare effect and characterized by the cosmic radiation being strongly anisotropic (the anisotropy being of amplitude $20-50 \%$ ), the direction of the anisotropy changing with time. Indirect arguments have suggested that this anisotropy is aligned parallel, or anti-parallel to the interplanetary magnetic field.

In this paper we examine the correlation between the observed direction of the cosmic ray anisotropy and the interplanetary magnetic field, and show directly that a major difference between the two classes of anisotropy is that the latter is field aligned, while the former is invariant with respect to magnetic field direction. We also discuss a flare effect during which the non-equilibrium anisotropy exhibited a persistent, anomalous phase, and show that this behaviour was closely correlated with the passage of a magnetic field sector boundary past the spacecraft.

The data considered here were obtained by the Pioneer VI spacecraft during the interval from 25 December, 1965 to May 18, 1966, during which time 20 solar flare effects were observed (Paper 1). The cosmic ray and magnetic field 
instrumentation have been described elsewhere (Bartley, McCracken and Rao, 1967; Ness, Scearce and Contarano, 1966). While both sets of instrumentation provide many directional samples each hour, we have, for the purpose of this paper, confined our attention primarily to hourly and 7.5 minute averages.

\section{THE ANISOTROPY VERSUS FIELD CORRELATION}

Figure 1 demonstrates the manner in which the cosmic ray anisotropy varied with time during a single flare effect. On the basis of the cosmic ray anisotropy data alone, we have defined two subsets of the totality of data obtained during large solar flares.

These subsets were defined as follows:-

Subset 1 . Those data obtained more than 6 hours after the commencement of a flare effect, for which the anisotropy amplitude was essentially invariant over a period of at least 12 hours, and for which the maximum cosmic ray flux was from the general direction of the sun. These selection criteria specify the "equilibrium anisotropy", as defined in Paper 1. Data illustrative of this subset are displayed in Figure 1.

Subset 2. This contained all solar flare data after the removal of subset 1 . This selection criterion specifies the non-equilibrium anisotropy as defined in Paper 1.

For each of the two subsets, the hourly average phase of the cosmic ray anisotropy was plotted against the concurrent hourly average direction of the interplanetary magnetic field projected into the plane of the ecliptic. (Figures 2 and 3). It is clear from Figure 2 (Subset 1) that while the cosmic ray anisotropy is always directed away from the sun during the "equilibrium" type anisotropy, there is no preferred direction of the magnetic field at such times. That is, the equilibrium anisotropy phase does not exhibit any correlation with the direction of the magnetic field vector. By contrast, there is a strong correlation between the anisotropy phase and the field direction in the case of the data in subset 2 (Figure 3). Note, however, that the regression coefficient between the directions of the cosmic ray anisotropy and the magnetic field is less than unity. A similar situation has been reported previously (Figure 1, McCracken and Ness, 1966).

It has been suggested elsewhere (McCracken, Rao, and Bukata, 1966) that the equilibrium anisotropy is due to the solar cosmic radiation population moving radially away from the sun at the solar wind velocity. The hypothesis would indicate, therefore, that the observed non-equilibrium anisotropy should be the sum of two components, one being an 


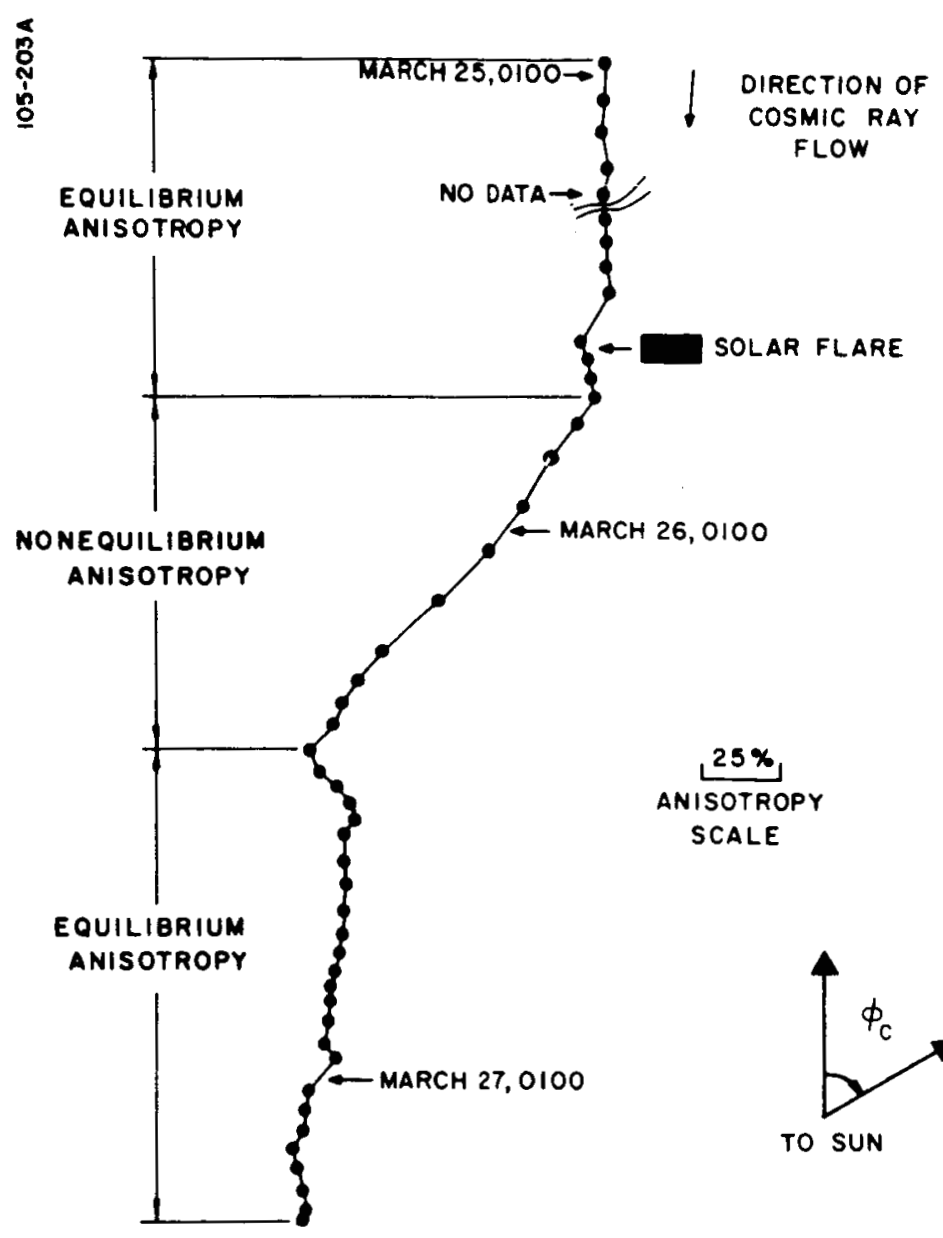

ANISOTROPY VECTOR DIAGRAM, MARCH 25-27, 1966

Figure 1-lllustrating the difference between the equilibrium andnon-equilibrium classes of cosmic ray ani sotropy. The amplitudes and azimuths of the mean ani sotropy for each hour are plotted as a vector addition diagram. Note definition of $\phi_{c}$. 

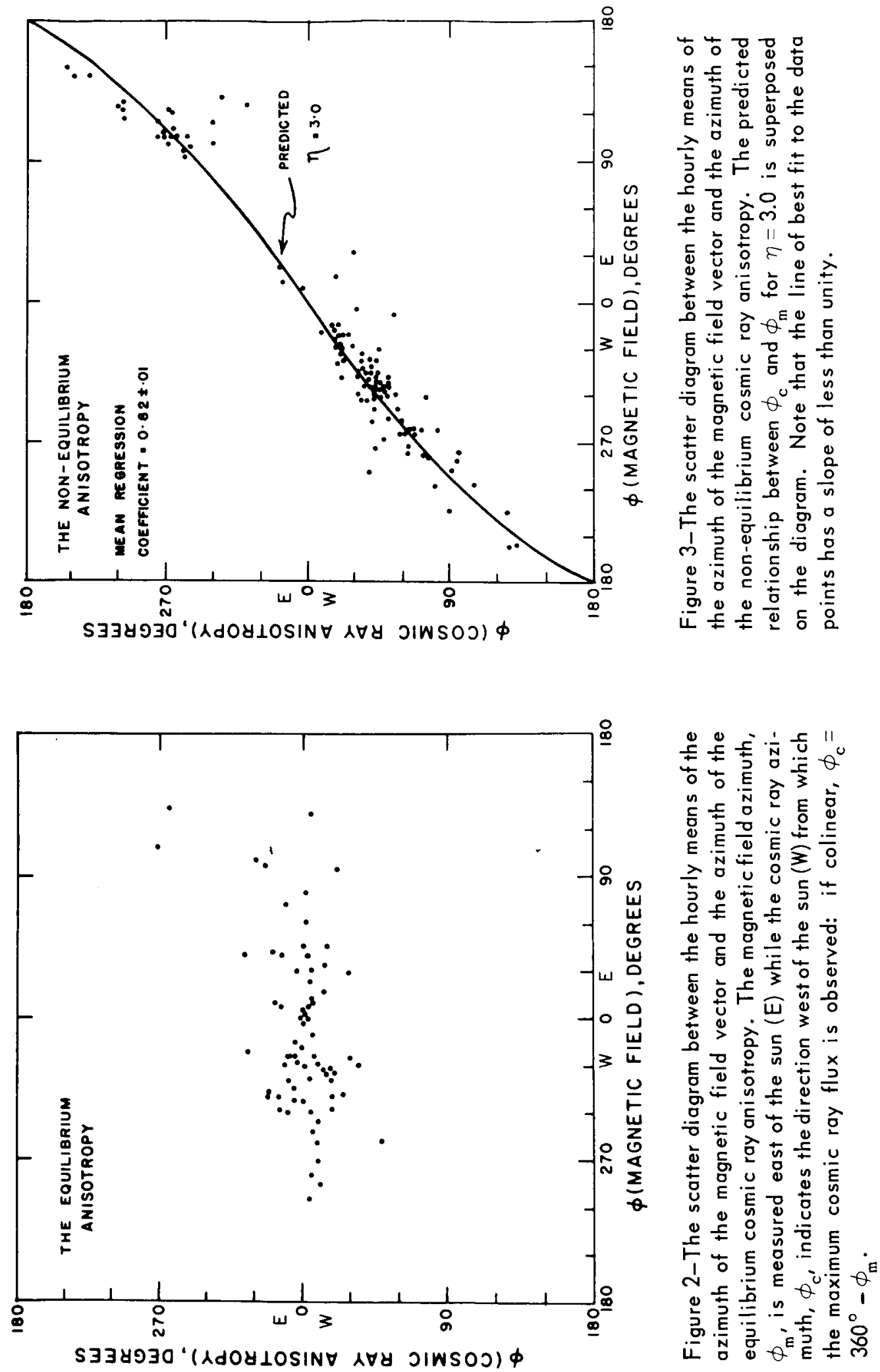
equilibrium type anisotropy, the other being a non-equilibrium anisotropy due to spatial inhomgeneities in the cosmic ray distribution in the solar system. This latter anisotropy would be oriented parallel, or antiparallel to the interplanetary magnetic field. Assuming a geometric situation such as depicted in Figure 4, in which the equilibrium anisotropy is directed radially away from the sun, and where $\phi_{m}$ is the azimuth of the interplanetary magnetic field, the amplitude and phase of the observed non-equilibrium cosmic ray anisotropy will given by

$$
\begin{aligned}
A_{T} & =A_{E} \sqrt{1+\eta^{2}+2 \eta \cos \phi_{m}} \\
\phi_{c} & =\arcsin \left\{\eta \sin \phi_{m}\left(1+\eta^{2}+2 \eta \cos \phi_{m}\right)^{-1 / 2}\right\} \\
\text { where } \eta & =A_{N} / A_{E}
\end{aligned}
$$

The first order approximation to equation (2) for small $\phi_{c}$ and $\phi_{m}$ can be written

$$
\phi_{\mathrm{c}}=\eta \cdot(1+\eta)^{-1} \cdot \phi_{\mathrm{m}}
$$

and consequently $\mathrm{d} \phi_{\mathrm{c}} / \mathrm{d} \phi_{\mathrm{m}}$, the theoretical regression coefficient of $\phi_{\mathrm{c}}$ upon $\phi_{\mathrm{m}}$, will approximate to $\eta(1+\eta)^{-1}$ for small $\phi_{\mathrm{m}}$. Consequently, the regression coefficient will always be less than unity. For example, the data presented in Figure 3 correspond to an observed mean amplitude of $25 \%$, while those in Figure 2 to a mean amplitude of $8 \%$, thereby implying $\eta \approx 3$ (on the assumption that the equilibrium anisotropy displayed in Figure 2 for late times during several flare effects is typical of the equilibrium anisotropies present at early times during the same flare effects). These figures imply $\mathrm{d} \phi_{\mathrm{c}} / \mathrm{d} \phi_{\mathrm{m}} \simeq 0.75$ for small $\phi_{\mathrm{m}}$. The relationship between $\phi_{\mathrm{c}}$ and $\phi_{\mathrm{m}}$ for all values of $\phi_{\mathrm{m}}$ is plotted in Figure 3 for $\eta=3$, and good agreement with the observed data is evident. We therefore conclude that the observed non-equilibrium anisotropies are explicable in terms of the superposition of a field aligned non-equilibrium anisotropy, and a radially directed equilibrium anisotropy.

All the solar flare observations of this paper are plotted in Figure 5 and it can be seen that there is still a pronounced correlation between the anisotropy 

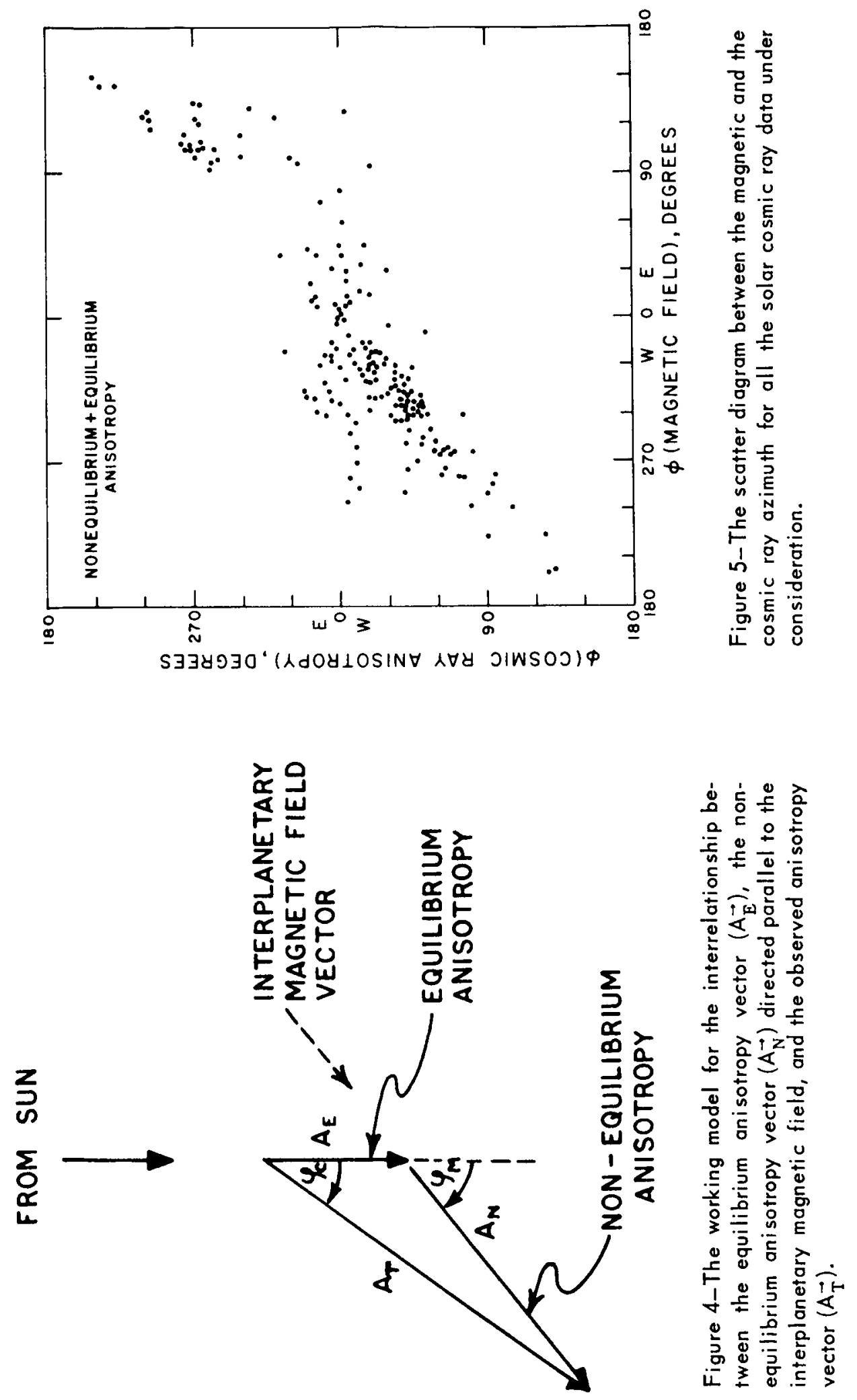
phase and the magnetic field direction. We note, however, that a regression analysis of data such as are presented in Figure 5 could lead to erroneous conclusions. Thus, writing the parameters of the population of data points which corresponds to the observed non-equilibrium anisotropy as unprimed quantities, and those of the equilibrium as primed quantities, we obtain the regression coefficient* of anisotropy direction $\left(\phi_{c}\right)$ upon magnetic field direction $\left(\phi_{m}\right)$ for the total population, $\mathrm{b}_{12}^{\mathrm{T}}$ to be

$$
\mathrm{b}_{12}^{\mathrm{T}}=\frac{\Sigma \phi_{\mathrm{c}} \phi_{\mathrm{m}}+\Sigma^{\prime} \phi_{\mathrm{c}}^{\prime} \cdot \phi_{\mathrm{m}}}{\Sigma \phi_{\mathrm{m}}{ }^{2}+\Sigma\left(\phi_{\mathrm{m}}^{\prime}\right)^{2}}
$$

and if $\mathrm{k}=\Sigma \phi_{\mathrm{m}}^{2} / \Sigma\left(\phi_{\mathrm{m}}^{\prime}\right)^{2}$ and assuming that

$b_{12}^{\prime}=0$ (as is suggested by Figure 2), then

$$
\mathrm{b}_{12}^{\mathrm{T}}=\mathrm{k}(1+\mathrm{k})^{-1} \mathrm{~b}_{12}
$$

and from (3)

$$
\mathrm{b}_{12}^{\mathrm{T}} \approx \mathrm{k} \eta(1+\mathrm{k})^{-1}(1+\eta)^{-1}
$$

where $\mathrm{k}$ is a measure of the relative importance of the non-equilibrium and equilibrium anisotropies in the data sample under consideration, and $b_{12}$ is the regression coefficient for the non-equilibrium anisotropy data alone. As an example, $\mathrm{k}=5$, and $\mathrm{b}_{12}=0.8$ for the data in Figure 5 , and hence the regression coefficient for the totality of the data is $b_{12}^{\mathrm{T}} \approx 0.67$.

From the above consideration it is clear that failure to recognise the existence of the two species of anisotropy will lead to the erroneous conclusion that the angle between the cosmic ray anisotropy direction and the interplanetary magnetic field vector is non-zero and is a function of the magnetic field direction.

\footnotetext{
*We stress here the fact that, in practice, neither the regression coefficient of $\phi_{c}$ upon $\phi_{m}$ ' nor $\phi_{\mathrm{m}}$ upon $\phi_{\mathrm{c}}$ are of physical significance, since both variates are subject to errors. The example given here is presented in the interests of simplicity; the same considerations apply to the more complicated but correct case in which the presence of errors in both variates is recognised explicitly in the mathematics. The qualitative conclusions are not affected by this simplification. Provided that the correlation coefficient between $\phi_{c}$ and $\phi_{m}$ is $\simeq 0.8$, a more correct version of equation (4) is obtained by replacing it by the mean $\mathrm{b}_{12}^{\mathrm{T}}$ of $\mathrm{b}_{12}^{\mathrm{T}}$ and $1 \mathrm{~b}_{21}^{\mathrm{T}}$. This latter approximation is correct to within $5 \%$ of the most rigorous curve of best fit.
} 
The foregoing observations very clearly demonstrate a fundamental difference between the equilibrium and non-equilibrium cosmic ray anisotropies. Since the same physical processes apply in interplanetary space, irrespective of the origin of the cosmic rays, we would expect to find both classes of anisotropy evident in the "galactic" cosmic radiation. In Paper 1 we have suggested that the "co-rotation" component of the diurnal variation (Axford (1965), Parker (1964)) belongs to the "equilibrium" class of cosmic ray anisotropies, while at least certain components of the "enhanced diurnal variation" belong to the non-equilibrium class. Clearly, a definite test of these hypotheses would be obtained by a detailed inter-comparison of the galactic cosmic ray anisotropy directions and the interplanetary magnetic field data. We would stress the need for extreme care in such analyses, however, due to the difficulty of separating the two classes of anisotropy on an a priori basis, and the resulting lack of knowledge of $\eta$ and $\mathrm{k}$ in equation (4).

\section{ANOMALOUS COSMIC RAY FLOW CHARACTERISTICS}

Examination of Figure 3 will show that there are a number of points corresponding to occasions on which the cosmic ray anisotropy phase was $>90^{\circ}$ and $<270^{\circ}$, indicative of there being an overall flow of solar cosmic rays towards the sun. Careful study has shown that every such point in Figure 3 corresponds to either the 30 December, 1965 or the April 29, 1966 flare effects (see Paper 1). The points for which the anisotropy phase is $<270^{\circ}$ correspond without exception to the flare effect of April 29, 1966 while the majority of the points for which the phase is $>90^{\circ}$ correspond to the December 30 flare effect. (This latter flare effect has been previously discussed in detail by Bartley et al (1966) and by McCracken and Ness (1966)).

Figure 6 displays the $7.5-45 \mathrm{MeV}$ proton counting rate, the cosmic ray anisotropy direction, and the direction of the interplanetary magnetic field at late times during the flare effect of April 29, 1966. It will be noted that changes in the anisotropy phase and the magnetic field direction were well correlated until at least $0900 \mathrm{UT}$ on April 30, and that the cosmic ray anisotropy was from directions other than the sun - spacecraft direction. That is, the anisotropy was of the non-equilibrium type until very late in the decay phase of the event. This in itself is unusual, since the cosmic ray anisotropy usually converts to an equilibrium anisotropy soon after the commencement of the decay phase of a flare effect. It should be noted that the flare effect of December 30 also exhibited very 


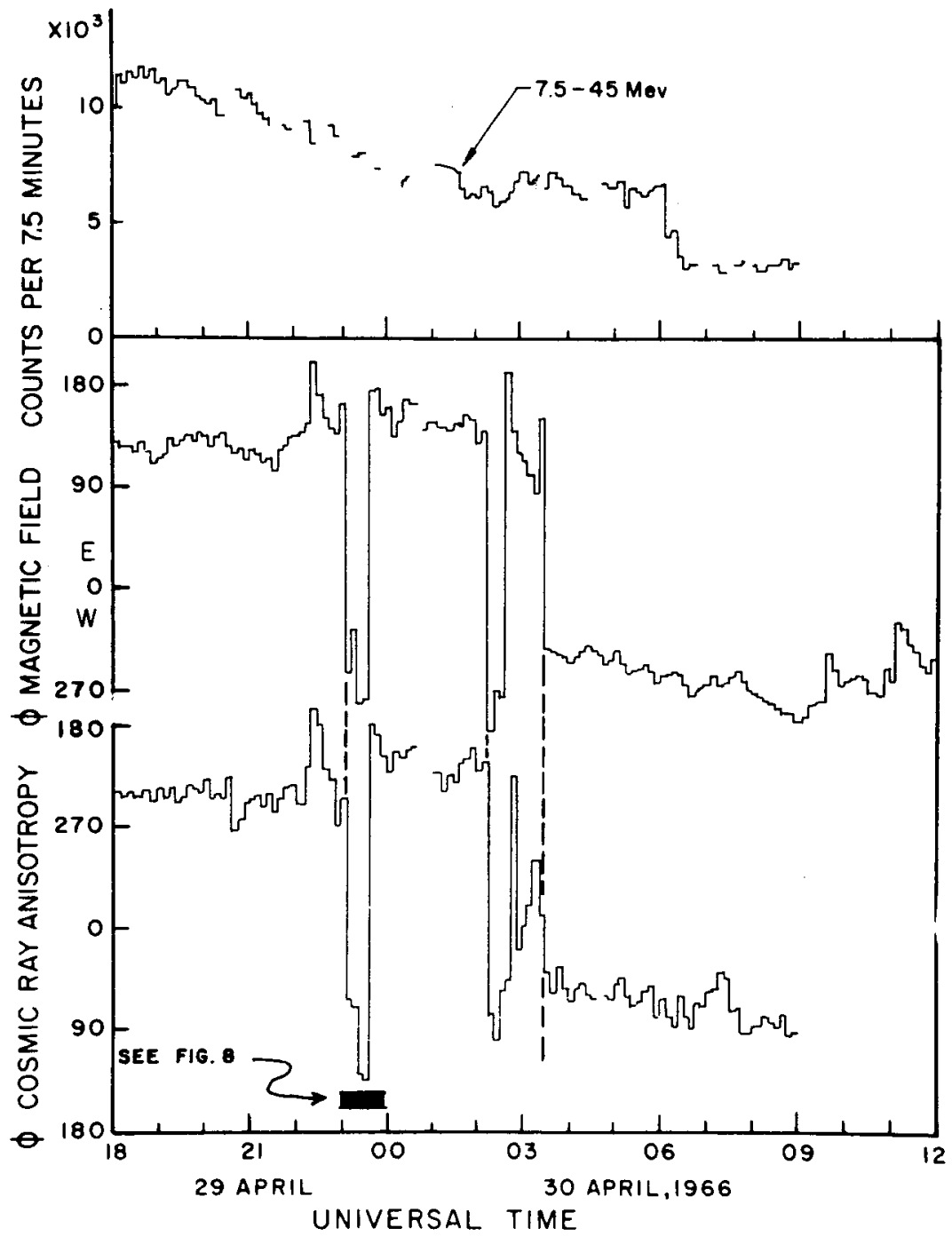

Figure 6-The temporal dependence of the flux of 7.5-45 Mev protons, of the azimuth of the magnetic field, and of the cosmic ray anisotropy, during the passage of a magnetic sector boundary past the spacecraft. Note the high degree of correlation between the changes in the magnetic and cosmic ray azimuths. 
long lived non-equilibrium anisotropies (Bartley et al, 1966). That is, both of the two flare effects which exhibited unusual anisotropy directions were also distinguished by virtue of their exhibition of very long lived non-equilibrium anisotropies.

Consideration of Figure 6 will show that throughout most of the period from $1800 \mathrm{UT}$ on 29 April to $0220 \mathrm{UT}$ on 30 April, the cosmic ray anisotropy was from an average direction of $125^{\circ}$ east of the sun. During this period of time the direction of the cosmic ray anisotropy agrees closely with the direction of the interplanetary magnetic field vector, that is, the maximum cosmic ray flux was streaming towards the sun along the lines of force of the interplanetary magnetic field. During the period 2300-2330 UT on 29 April, and after 0300 UT on 30 April, however, both the cosmic ray anisotropy and the magnetic vector exhibited directions which differ by $180^{\circ}$ from the earlier value. That is, during these latter intervals, the solar cosmic radiation was streaming away from the sun along the lines of force of the interplanetary magnetic field. Inspection of the magnetic field data for the days prior and subsequent to those in Figure 6 indicates that the abrupt reversals of the field direction in Figure 6 were part of a series of abrupt field reversals associated with the passage of a magnetic sector boundary (Wilcox and Ness, 1965) past the spacecraft. That is, in the vicinity of the sector boundary the cosmic ray streaming direction exhibited $180^{\circ}$ phase reversals in synchronism with the magnetic field reversals.

For a field-aligned anisotropy the direction from which the anisotropic flux of cosmic radiation is streaming indicates the direction that leads, via a field line, from the point of observation to the vicinity of the solar region that generated the solar cosmic radiation. That is, it indicates the direction to the sun insofar as a charged particle is concerned. Thus, while careful examination of solar flare records (CRPL Reports, 1966) has failed to identify a parent solar flare responsible for the solar proton event on April 29/30, the anisotropy direction indicates that subsequent to 0300 UT the spacecraft was probably connected to the vicinity of the source region via a simple Archimedes spiral field line. (See Figure 7). Prior to $0300 \mathrm{UT}$, however, the anomalous anisotropy direction indicates that the field line direction away from the sun made a connection with the vicinity of the source region on the sun. The absence of any marked discontinuity in the solar cosmic ray intensity versus time curve in the vicinity of the field reversals (Figure 6) indicates that points on either side of the field reversals were both equally favorably situated relative to the source region, and that, in particular, they were both roughly equal distances from the source region (along the magnetic lines of force). That is, the cosmic ray and magnetic field data suggest that the lines of force observed prior to crossing a field reversal connected to the vicinity of the source region, the field line changing direction (i.e. - exhibiting a "loop") in the vicinity of 1.A.U. (See Figure 7). An alternative 


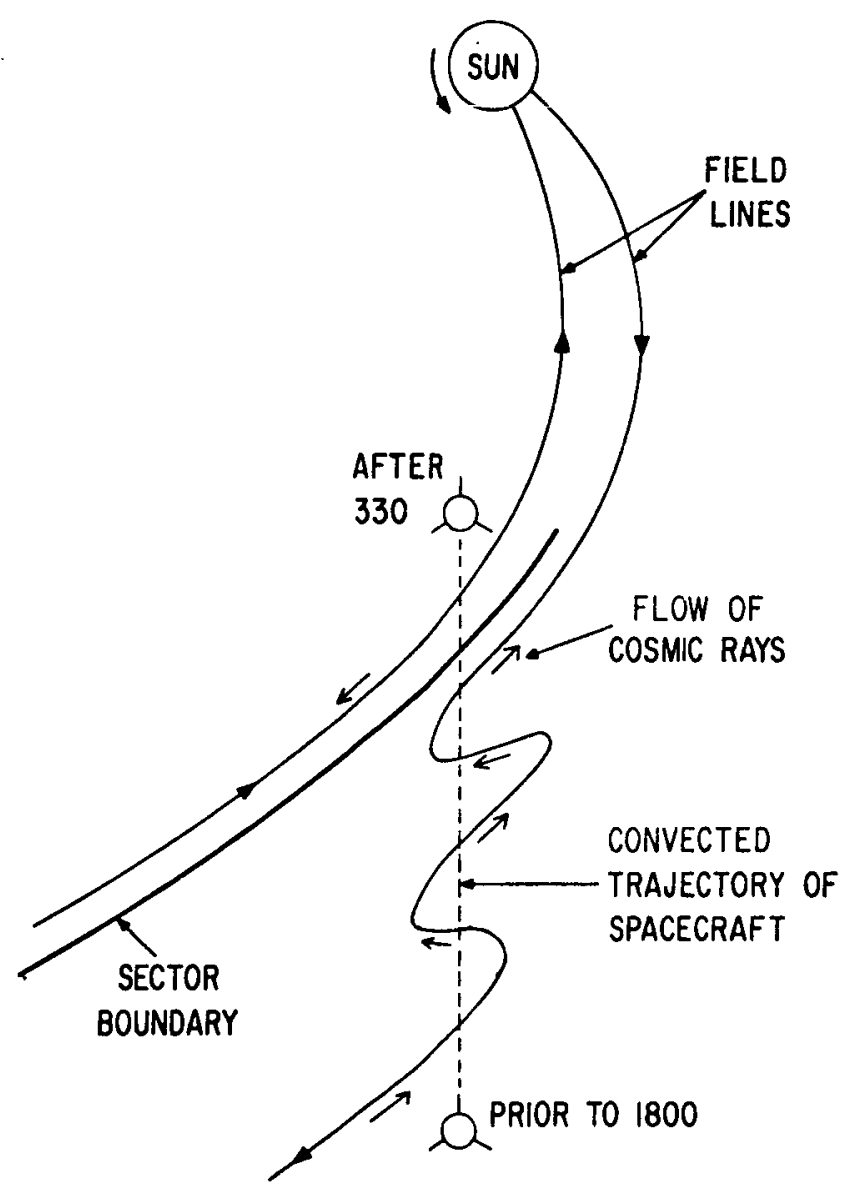

Figure 7-lllustrating one model proposed to explain the simultaneous reversals of the magnetic and cosmic ray ani sotropy azimuths. The positions of the Pioneer VI spacecraft relative to the interplanetary magnetic field ot times prior, and subsequent to 0300 UT are shown. 
possibility is that the sequence of field reversals is related to the occurence of two filaments in the interplanetary field, both of which are rooted to the same source region as the fields on opposite sides of the sector boundary and which also possess complex loops.

Figure 8 presents detailed cosmic ray anisotropy phase and amplitude data for the period 2245-2345 UT, this interval being indicated in Figure 6 by the cross-hatched box. Each graphical point corresponds to data accumulation over a period of 56 seconds, this being the repetition rate for data transmission for the cosmic ray experiment on the spacecraft at the time in question. That is, these data illustrate the maximum time resolution obtainable from the experiment during this phase of the flight of the spacecraft.

From Figure 8 , it is clear that the reversals of the direction of the cosmic ray anisotropy evident in Figure 6 occurred within a time scale $\tau \approx 56$ seconds. Indicating the gyro radius of a $11 \mathrm{Mev}$ proton (the mean energy for the data in Figure 8) by $\rho$, the cosmic ray pitch angle by $\xi$, the Archimedes spiral angle by $\phi_{m}$, and the plasma velocity by $v_{p}$, then the completion of the reversal of the cosmic ray anisotropy in a time comparable to $\tau$ implies.

$$
\begin{array}{r}
\mathrm{v}_{\mathrm{p}} \cdot \tau \gtrsim 2 \rho \sin \xi / \mathrm{sin} \phi_{\mathrm{m}} \\
\text { or sin } \xi \leq \frac{\mathrm{v}_{\mathrm{p}} \cdot \tau}{2 \rho} \sin \phi_{\mathrm{m}} .
\end{array}
$$

where it is assumed that the observer has to be further than $\rho \sin \xi$ from the interface to see cosmic radiation unaffected by the adjacent particle stream. For typical values of $v_{p} \approx 435 \mathrm{~km} \mathrm{sec}{ }^{-1} ; \rho \approx 10^{5} \mathrm{~km}$ (which corresponds to a $11 \mathrm{Mev}$ proton in a $5 \times 10^{-5}$ oe field); and $\phi_{\mathrm{m}}=45^{\circ}$, then

$$
5 \leqslant 5^{\circ}
$$

That is, the anisotropic component of the solar cosmic radiation near the field reversals was strongly collimated, indicative of there being little scattering of the cosmic rays, despite their proximity to the field reversal.

The flare effect of December 30 continued until January 1, 1966, throughout which time a variable, field aligned anisotropy was observed, some of the anisotropy phases implying cosmic ray flow towards the sun. (Bartley et al, 1966; McCracken and Ness, 1966). A magnetic sector boundary passed by the spacecraft early on January 2 , by which time the solar cosmic ray fluxes were too 


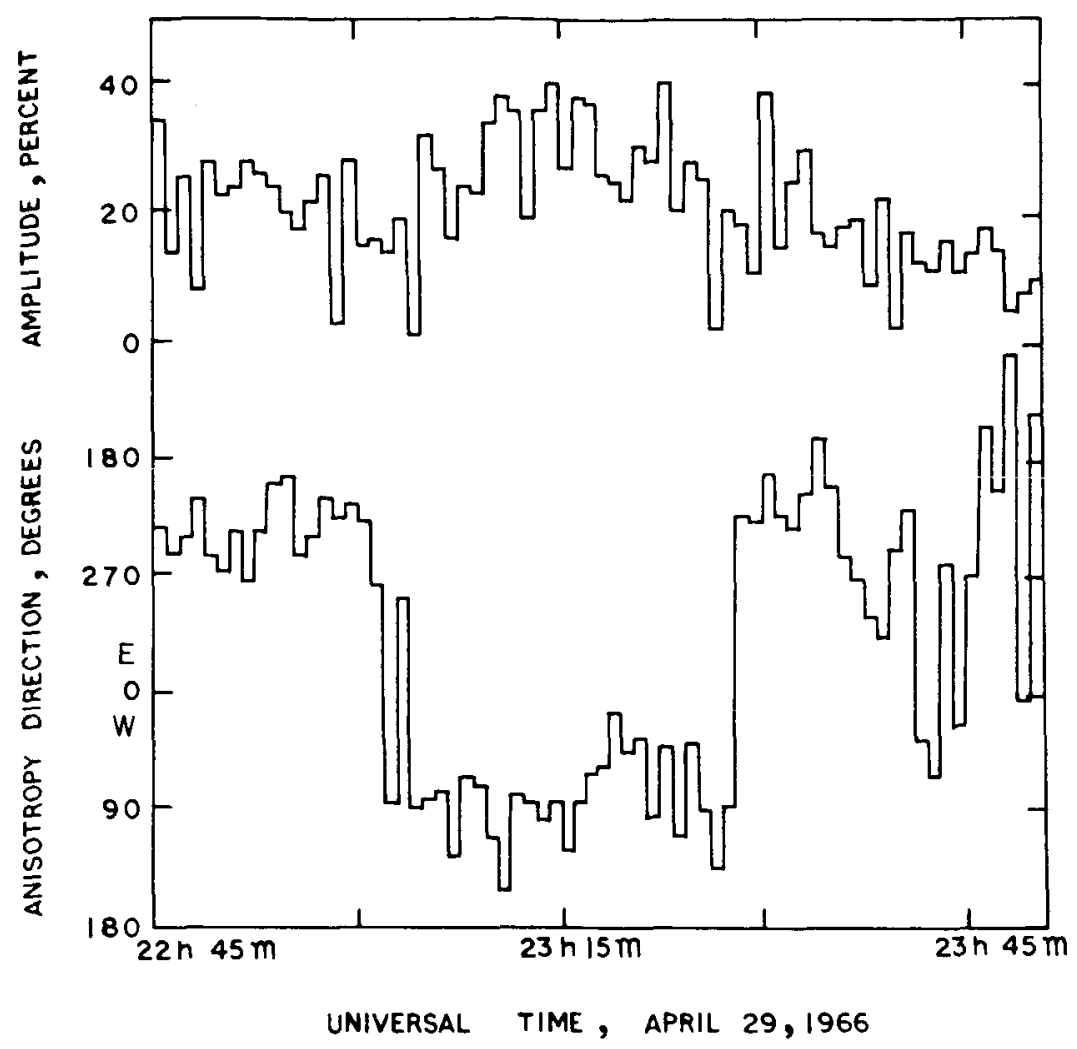

Figure 8-The detailed temporal dependence of the amplitude and azimuth of the cosmic ray anisotropy during the field and anisotropy reversal indicated in Fig. 6 by the cross-hatched box. Note the abrupt nature of the reversals of the anisotropy azimuth.

small to permit definite study. However, this event and the event of April 29 certainly exhibit the following characteristics in common.

(a) Long persistent non-equilibrium anisotropies ( $~(12$ hours duration).

(b) Apparent solar cosmic ray flow towards the sun, the sunward flow sometimes persisting for many hours.

(c) Proximity to the passage of a magnetic sector past the spacecraft.

(d) Extremely rapid changes ( $\tau \approx 56 \mathrm{sec}$ ) in the direction of the cosmic ray anisotropy.

Characteristics (a) and (b) have not been observed in other flare effects, and upon these characteristics alone these two proton events are deemed to be unique, and 
similar to one another. The evidence, and in particular the reversal in phase of the cosmic ray anisotropy on April 30 at the time of the sector passage past the spacecraft suggests that the unusual characteristics outlined above were due to the spacecraft being in the proximity of a sector boundary at the time the flare occurred.

\section{CONCLUSIONS}

On the basis of the direct evidence reviewed herein, and in Paper 1, we conclude that the two species of cosmic ray anisotropy defined in Paper 1, exhibit the following characteristics:-

The Equilibrium Anisotropy is field invariant and the phase and amplitude are invariant with respect to time. This species of anisotropy is an indicator of a cosmic ray distribution in the solar system which is in diffusive equilibrium. The equilibrium anisotropy is aligned parallel with the solar wind velocity vector.

The Non-Equilibrium Anisotropy is field aligned; it may change markedly in direction and amplitude with time; and is an indicator of a non-homogeneous distribution of cosmic rays in the solar system. Under such circumstances, the redistribution of cosmic rays throughout the solar system will occur preferentially along the interplanetary magnetic field lines.

In addition, there is evidence suggesting a tendency for the observation of non-equilibrium anisotropies over an extended period of time if the spacecraft is near the boundary of a magnetic sector. The data indicate that near the sector boundary observed by Pioneer VI on April 30, 1966, there was a direct connection between the lines of force on either side of the boundary to the source region of the cosmic ray particles. The manner in which the anisotropy changes upon crossing the boundary indicates strongly collimated (pitch angles $\lesssim 10^{\circ}$ ) radiation on both sides of the boundary.

\section{ACKNOWLEDGEMENT}

The cosmic ray detector was developed at the Southwest Center for Advanced Studies, Dallas, Texas, K. G. McCracken and U. R. Rao are indebted to Mr. W. C. Bartley and Dr. R. P. Bukata for help of many kinds during the development, construction, integration and pre-flight check-out of the detector. A portion of the initial data processing was also accomplished at SCAS, and the assistance of Messrs. C. Peters and R. Lehman is gratefully acknowledged. 
This research was supported by the National Aeronautics and Space Administration under contract NSR-44-004-003, by AFCRL contract AF 19 (628)-5039, and by funds provided by the Indian Atomic Energy Commission. The magnetic field instrument was developed at NASA-GSFC by S. Cantarano, C. Ehrmann and C. S. Scearce. Discussions with Drs. L. F. Burlaga and J. M. Wilcox are appreciated.

\section{REFERENCES}

1. Axford, W.I.; "Anisotropic Diffusion of Solar Cosmic Rays"; Planet. Space Sci., 13, 1301-1309, 1965.

2. Bartley, W. C., R. P. Bukata, K. G. McCracken and U. R. Rao; "Anisotropic Cosmic Radiation Fluxes of Solar Origin"; J. Geophys. Research, 71, $3297-$ $3304,1966$.

3. Bartley, W. C., K. G. McCracken and U. R. Rao; "The Pioneer VI detector to measure the degree of anisotropy of the cosmic radiation in the energy range 7.5 - $90 \mathrm{Mev} /$ nucleon"; Rev. Sci. Instr; 38, 266-272, 1967.

4. Mc Cracken, K. G., and N. F. Ness; The Collimation of Cosmic Rays by the Interplanetary Magnetic Field"; J. Geophys. Research, 41, 3315-3318, 1966.

5. McCracken, K. G., U. R. Rao and R. P. Bukata; "Cosmic Ray Propagation Processes; 1. A Study of the Cosmic Ray Flare Effect"'; J. Geophys. Research, 72, 4293-4324, 1967.

6. Ness, N. F., C. S. Scearce and S. Cantarano; "Preliminary Results from the Pioneer VI Magnetic Field Instrument"; J. Geophys. Research; 71, 3305$3313,1967$.

7. Parker, E. N.; "Theory of streaming of cosmic rays and the dirunal variation"; Planet. Space Sci., 12, 735-747, 1964.

8. Wilcox, J. M., and N. F. Ness; "Quasi-stationary Corotating structure in the interplanetary medium"; J. Geophys. Research 70, 5793-5805, 1965. 